\title{
Arbor
}

\section{eEurope y el sector de la electrónica y las comunicaciones}

\section{Erkki Liikanen}

Arbor CLXXV, 690 (Junio 2003), 819-825 pp.

En el año 2000, la estrategia definida en la Cumbre de Lisboa se basó en el reconocimiento de que Internet es una fuente importante de crecimiento y productividad.

Este fue el fundamento sobre el que se construyó el Plan de Acción eEurope 2002, que llegó a su término en diciembre de ese año. La Comisión publicó posteriormente su informe final de eEurope 2002. Desde el 2000, el número de usuarios residenciales conectados se ha más que doblado, y prácticamente todas las escuelas y empresas están ahora online. Un nuevo y moderno marco legal para los servicios de comunicaciones y e-commerce fue adoptado a escala europea. eEurope, contribuyendo a ello, ha demostrado su éxito.

Ahora debemos mirar adelante y orientar a Europa hacia Internet de alta velocidad y acceso multiplataforma. El ancho de banda cambia radicalmente el uso de Internet y mejora sus capacidades. Los ciudadanos pueden acceder a la banda ancha y beneficiarse de ella a través de nuevas plataformas de comunicación como la TV digital interactiva y la telefonía móvil de tercera generación.

El Plan de Acción eEurope 2005 nos acerca al objetivo de conseguir una sociedad de la información para todos. eEurope 2005 pone el mayor énfasis en acelerar el desarrollo de las infraestructuras de banda ancha, al mismo tiempo que en la creación de servicios interactivos multimedia más atractivos.

En una situación como la actual eEurope 2005 jugará un papel crucial en la restauración de la confianza en el sector de la electrónica y las comunicaciones. En concreto los beneficios sociales y económicos que se pueden esperar de la banda ancha y la tecnología 3G hace 
más urgente que nunca la necesidad de mejorar la actual disponibilidad y uso de estas tecnologías. Más adelante revisaremos las principales acciones identificadas por la Comisión para alcanzar estos objetivos.

\section{De eEurope 2002 a eEurope 2005}

La mayoría de los 64 objetivos del Plan de Acción eEurope han sido alcanzados. Ello se debe al esfuerzo de muchas personas en las instituciones europeas, estados miembros, la industria y los agentes sociales. El logro es particularmente notable si nos damos cuenta de que ha sido alcanzado a pesar del difícil entorno económico y la caída generalizada de los mercados bursátiles.

Tres datos pueden resumirlo mejor:

- El acceso a Internet se ha más que doblado, con el $43 \%$ de los usuarios residenciales conectados en noviembre de 2002.

- En el 2002, más del $90 \%$ de escuelas y empresas estaban conectadas a Internet.

- Europa dispone en la actualidad de la red troncal de investigación más rápida del mundo, accesible por 32 países.

eEurope ha jugado un papel crucial como catalizador de la política adoptada por la Unión Europea mediante la introducción de modificaciones regulatorias que han mejorado rotundamente el entorno legal para el desarrollo de los servicios de comunicaciones y el comercio electrónico.

Europa está mejor situada que nunca para aprovechar los beneficios de Internet y la tecnología digital, y traducirlos en mejora de la productividad, crecimiento económico, empleo y cohesión social. Y la situación puede todavía mejorar.

Pero los beneficios económicos, el incremento de la productividad, la mejora de la calidad de los servicios, la cohesión social y el crecimiento sin inflación no llegan por sí solos. Para lograrlo, hay que introducir Internet en nuestra vida, reestructurando nuestros modelos económicos, modernizando métodos, adquiriendo nuevas habilidades y reorientando el cambio organizativo de manera que aproveche a fondo los beneficios de las nuevas tecnologías. Y eso toma tiempo. 
El reto, pues, es estimular el uso de Internet:

- La conectividad en las escuelas ha sido ya alcanzada. Ahora, el mayor énfasis hay que ponerlo en disponer de productos y servicios e-learning de calidad, que puedan ser utilizados en un contexto pedagógico adecuado. Necesitamos contenidos de banda ancha, maestros entrenados y organizaciones adaptadas. Lo que significa nuevas interacciones sociales dentro y fuera de las escuelas.

- Para alcanzar el máximo de eficiencia, la pequeña y mediana empresa precisa integrar estas tecnologías en sus esquemas habituales de negocio. Además, las grandes compañías se lo van a solicitar como parte de la relación comercial, pues ya disponen de estas herramientas.

- Todavía hay un gran potencial de desarrollo de servicios de gobierno electrónico, aunque los estados miembros han progresado de forma importante los últimos años. Los servicios básicos de la Administración son ya online. Ahora debe aumentarse el grado de hiperactividad, lo que probablemente requerirá reorganizaciones internas para conseguir la necesaria eficiencia.

La nueva etapa de desarrollo de la sociedad de la información, en la línea de los objetivos de Lisboa, ya ha sido definida en el Plan de Acción eEurope 2005, que cubre el período 2003/2005.

El nuevo Plan de Acción está enfocado a un menor número de objetivos, pero de cuya consecución puede obtenerse una significativa mejora: la modernización de los servicios públicos para mejorar su productividad, accesibilidad y equitativa distribución; la promoción de entornos favorables para el comercio electrónico y una infraestructura de banda ancha multiplataforma que sea segura. Detrás de todo esto se puede reconocer fácilmente el empeño por crear una sociedad de la información para todos los ciudadanos europeos.

Vamos a describir estas acciones con mayor detalle

\section{Servicios, aplicaciones y contenidos:}

Ahora que el acceso a Internet es algo generalizado, es necesario desarrollar los servicios, aplicaciones y contenidos que aprovechen este potencial para el progreso humano y económico. eEurope 2005 está 
enfocado principalmente a la administración, educación, salud y comercio electrónicos.

Nuestra intención principal es modernizar los servicios públicos en el sentido de hacerlos más productivos, eficientes, equitativos y fáciles de usar. Para conseguirlo será clave que los servicios públicos esenciales estén disponibles online, invertir en recursos humanos y una completa reingeniería de la administración pública.

En paralelo revisaremos la legisláción que afecta al comercio electrónico. La intención es identificar y posteriormente eliminar los elementos legales que retrasan o impiden el desarrollo del comercio electrónico y la generalización de modelos de negocio innovadores, especialmente en la PYME. También es importante a este respecto fomentar la confianza del consumidor.

\section{El desarrollo de una infraestructura de banda ancha segura:}

Las comunicaciones electrónicas han crecido exponencialmente en los últimos años. Esta expansión afecta a todos los sectores de la actividad humana; ya sean negocios, servicios públicos, o la esfera privada. Como resultado de todo esto, las redes globales han llegado a ser vitales para nuestras sociedades y economías.

Hoy Internet es sólo una pequeña muestra de lo que la Internet del mañana, totalmente multimedia, será, cuando la nueva generación de servicios y aplicaciones lleguen al mercado. Lo que dependerá del desarrollo acelerado de infraestructuras de banda ancha por cable o radio que sean accesibles para todos.

La Comisión instituyó recientemente el «Día de la Banda Ancha» con los estados miembros y la industria, lo que demuestra que el reto sobre todo es disponer cuanto antes de estos recursos.

Adaptar las infraestructuras y desarrollar nuevas redes es, en primer lugar, una tarea del mercado. Los gobiernos pueden a su vez facilitar la inversión privada mediante la propia demanda y actuando en la eliminación de las barreras legislativas y administrativas.

La infraestructura de banda ancha debe ser totalmente segura. La Comisión ha puesto en marcha recientemente una Agencia Europea de Seguridad de Red e Información. Será un centro de competencia y de coordinación de esfuerzos nacionales para aumentar la seguridad de la información y de la red de los estados miembros y de las instituciones de la Unión, que sea capaz de prevenir y responder a todas las amenazas y problemas en este sentido. 
Una mayor seguridad contribuirá a aumentar la confianza en Internet del consumidor, y este es un objetivo importante dentro de la estrategia de la Comisión, lo que incluye medidas tendientes a promocionar códigos de conducta y sistemas flexibles de resolución de disputas.

\section{Acceso para todos:}

De todo lo anteriormente mencionado se extrae una idea común: crear la sociedad de la información para todos. Esto significa asegurar la inclusión de los social o geográficamente marginados, así como la accesibilidad para las personas con necesidades especiales, como los discapacitados y enfermos.

Este objetivo final es la guía para la implementación de eEurope 2005. Por ejemplo, se da un papel fundamental a las iniciativas locales en las áreas remotas o menos pobladas.

Además se toma muy en cuenta las experiencias en los niveles más cercanos al usuario, ya sea regiones, municipalidades, escuelas, bibliotecas, hospitales, etc.

\section{El Estado del sector:}

Entonces, ¿cuál es la actual situación del sector de las telecomunicaciones electrónicas?. La Comisión ha estado monitorizando permanentemente su evolución. Como un panorama de situación, en respuesta a la pregunta del Consejo de Telecomunicaciones del 5 de diciembre de 2002, la Comisión ha hecho suyo recientemente una Comunicación titulada «El camino hacia la economía del conocimiento» nuestro más inmediato reto es restaurar el clima de confianza necesario para la inversión en banda ancha y $3 \mathrm{G}$.

Nuestra intención es presentar una estrategia coherente y sugerir acciones para implementarla. A los estados miembros se les recuerda la necesidad de completar rápidamente el proceso de definición e implementación de las líneas de actuación ya planificadas y comprometidas. Banda ancha y $3 G$ son áreas en las que la inversión está actualmente parada a causa de la situación financiera general del sector. Las barreras para el desarrollo de redes han de ser eliminadas. Este es el momento adecuado para insistir en la necesidad de armonizar los esfuerzos para superar la fragmentación de los mercados nacionales 
en comunicaciones móviles. Además dado el interés que los estados miembros han demostrado en nuestro reciente Día de la Banda Ancha, la Comunicación propone un compromiso para adaptar las estrategias nacionales a objetivos definidos.

Hay tres tipos de acciones relacionadas:

1. En primer lugar una completa y efectiva implementación del nuevo marco regulatorio para las telecomunicaciones electrónicas. Este nuevo marco legal, vigente desde julio de 2003 en todos los estados miembros creará el entorno regulatorio estable y predecible que necesitan las compañías telefónicas, operadores de cable, emisoras de televisión digital, compañías móviles y de satélite, etc.

Desde principios de 2000 la Comisión ultimó los detalles de este nuevo marco regulatorio con la adopción de su Recomendación para los mercados relevantes.

2. La segunda categoría coordina las acciones dirigidas a fomentar el uso de los contenidos, servicios, y aplicaciones a través de la banda ancha y el acceso multiplataforma, tal y como está definido en el Plan de Acción eEurope 2005.

Ello incluye la puesta en marcha de estrategias nacionales de banda ancha con hitos claros para finales de 2003; así como la supresión de barreras para el desarrollo de 3G. El objetivo debería consistir en que la mitad de las conexiones a Internet en el 2005 sean de banda ancha. También las actuaciones deberían ir encaminadas a acelerar la disponibilidad de servicios y aplicaciones de gobierno, salud y educación electrónica.

3. En tercer lugar, pero no menos importante, estarían las actuaciones destinadas a incrementar el actual esfuerzo de investigación tanto a escala nacional como de la Unión Europea; de manera que se asegure la competitividad a largo plazo.

Se puede resumir esta triple estrategia de la siguiente manera: para alcanzar el liderazgo en el sector de las comunicaciones electrónicas, es necesario disponer de las condiciones legales que lo posibiliten. Además la demanda y la fabricación deben ser estimuladas, de manera 
que el negocio aumente y los consumidores puedan acceder a los beneficios de la tecnología. Finalmente, invertir en I+D es imprescindible para asegurar a largo plazo el sector.

Esta estrategia fue adoptada por los estados miembros en el Consejo Europeo de Bruselas de marzo de 2003.

\section{Conclusión}

La sociedad de la información depende de la voluntad de trabajar juntos de los diferentes estamentos y empresas. Con eEurope 2005 creemos haber definido el camino correcto que permitirá restaurar la confianza en el sector. 